\title{
Organizational Contact Telecom Address
}

National Cancer Institute

\section{Source}

National Cancer Institute. Organizational Contact Telecom Address. NCI Thesaurus.

Code C94264.

A sequence of digits or characters used to identify a particular telephone, fax, or email of the organizational contact. 